ACCEPTED MANUSCRIPT

\title{
Flexible deep-ultraviolet light-emitting diodes for significant improvement of quantum efficiencies by external bending
}

To cite this article before publication: Shahab Shervin et al 2018 J. Phys. D: Appl. Phys. in press https://doi.org/10.1088/1361-6463/aaaabf

\section{Manuscript version: Accepted Manuscript}

Accepted Manuscript is "the version of the article accepted for publication including all changes made as a result of the peer review process, and which may also include the addition to the article by IOP Publishing of a header, an article ID, a cover sheet and/or an 'Accepted

Manuscript' watermark, but excluding any other editing, typesetting or other changes made by IOP Publishing and/or its licensors"

This Accepted Manuscript is @ 2018 IOP Publishing Ltd.

During the embargo period (the 12 month period from the publication of the Version of Record of this article), the Accepted Manuscript is fully protected by copyright and cannot be reused or reposted elsewhere.

As the Version of Record of this article is going to be / has been published on a subscription basis, this Accepted Manuscript is available for reuse under a CC BY-NC-ND 3.0 licence after the 12 month embargo period.

After the embargo period, everyone is permitted to use copy and redistribute this article for non-commercial purposes only, provided that they adhere to all the terms of the licence https://creativecommons.org/licences/by-nc-nd/3.0

Although reasonable endeavours have been taken to obtain all necessary permissions from third parties to include their copyrighted content within this article, their full citation and copyright line may not be present in this Accepted Manuscript version. Before using any content from this article, please refer to the Version of Record on IOPscience once published for full citation and copyright details, as permissions will likely be required. All third party content is fully copyright protected, unless specifically stated otherwise in the figure caption in the Version of Record.

View the article online for updates and enhancements. 


\title{
Flexible deep-ultraviolet light-emitting diodes for significant improvement of quantum efficiencies by external bending
}

\author{
Shahab Shervin, ${ }^{1}$ Seung Kyu Oh, ${ }^{1,3,9}$ Hyun Jung Park, ${ }^{3}$ Keon-Hwa Lee, ${ }^{1,4}$ Mojtaba Asadirad, ${ }^{1}$ Seung- \\ Hwan Kim, ${ }^{1,5}$ Jeomoh Kim, ${ }^{6}$ Sara Pouladi, ${ }^{2}$ Sung-Nam Lee, ${ }^{7}$ Xiaohang Li,${ }^{8}$ Joon Seop Kwak, ${ }^{3, b)}$ and Jae- \\ Hyun Ryou ${ }^{1,2,9, a)}$ \\ ${ }^{1}$ Department of Mechanical Engineering, University of Houston, Houston, Texas 77204-4006, USA \\ ${ }^{2}$ Materials Science and Engineering Program, University of Houston, Houston, Texas 77204, USA \\ ${ }^{3}$ Department of Printed Electronics Engineering, Sunchon National University, Suncheon, Jeollanam-do, \\ 57922, Korea \\ ${ }^{4}$ Korea Photonics Technology Institute (KOPTI), Gwangju, 61007, Korea \\ ${ }^{5}$ Metamaterial Electronic Device Research Center, Hongik University, Seoul, 04066, Korea \\ ${ }^{6}$ LG Electronics, Seoul, 06763, Korea \\ ${ }^{7}$ Department of Nano-Optical Engineering, Korea Polytechnic University, Siheung, Gyeonggi-do, 15073, \\ Korea \\ ${ }^{8}$ Computer, Electrical and Mathematical Sciences and Engineering (CEMSE) Division, King Abdullah \\ University of Science and Technology (KAUST), Thuwal, 23955, Saudi Arabia \\ ${ }^{9}$ Texas Center for Superconductivity at the University of Houston (TcSUH) and Advanced \\ Manufacturing Institute (AMI), University of Houston, Houston, Texas 77204, USA
}

a) Electronic mail: jryou@uh.edu

a) Electronic mail: jskak@sunchoh.ac.kr

\begin{abstract}
We report a new route to improve quantum efficiencies of AlGaN-based deep-ultraviolet lightemitting diodes (DUV LEDs) using mechanical flexibility of recently developed bendable thin-film structures. Numerical studies show that electronic band structures of AlGaN heterostructures and resulting optical and electrical characteristics of the devices can be significantly modified by external bending through active control of piezoelectric polarization. Internal quantum efficiency (IQE) is enhanced higher than three times, when the DUV LEDs are moderately bent with concave curvatures. Furthermore, efficiency droop at high injection currents is mitigated and turn-on voltage of diodes decreases with the


same bending condition. The concept of bendable DUV LEDs with a controlled external strain can provide a new path for high-output-power and high-efficiency devices.

Keywords: deep ultraviolet (DUV), light-emitting diodes (LEDs), quantum efficiency, flexible electronics

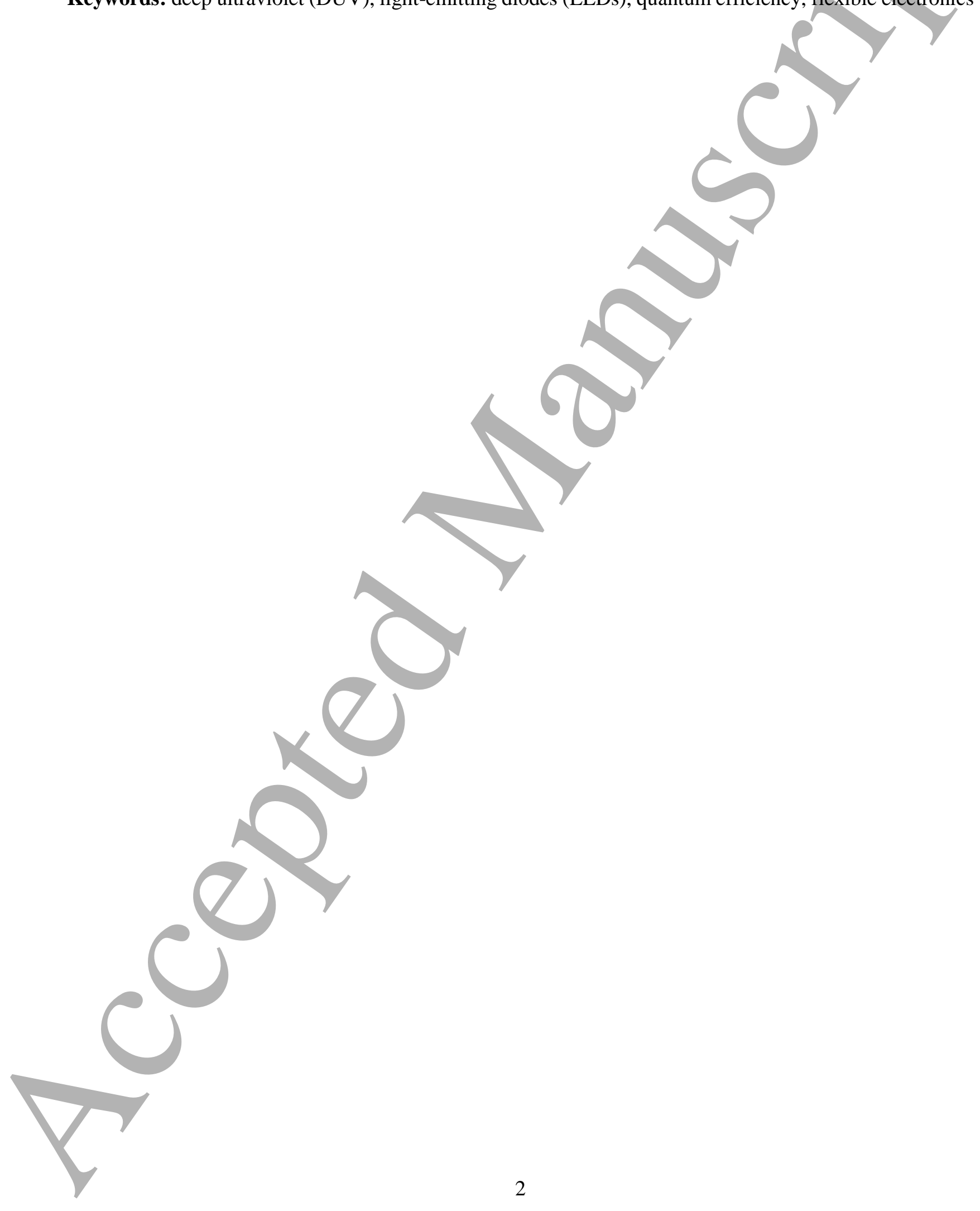




\section{Introduction}

Deep-ultraviolet (DUV) photons with wavelengths $\lambda<280 \mathrm{~nm}$ are useful for a number of applications in sterilization, water- and air-purification, chemical/biochemical detection, gas sensors, photolithography, non-line-of-sight communication [1]. Current conventional DUV light sources, gasdischarged lamps are bulky and fragile, and have short lifetimes. Also, widely-used mercury lamps contain toxic element that raises environmental and health concerns. Light-emitting diodes (LEDs) based on $\mathrm{Al}_{\mathrm{x}} \mathrm{Ga}_{1-\mathrm{x}} \mathrm{N}$ semiconductor heterostructures have the potential to offer efficient, compact, reliable, and environment-friendly DUV light sources with similar beneficial characteristics to their siblings, visible LEDs. However, the current status of DUV LEDs operating at $\lambda=240-280 \mathrm{~nm}$ is far less matured than that of the visible LEDs. The overall efficiency, i.e., external quantum efficiency (EQE) is generally less than $10 \%$ [2]. These EQEs are not only significantly lower than those of the blue LEDs ( 80\%) [3], but also lower than those of the gas-discharged lamps $(\sim 30 \%)$. High density of the defects such as threading dislocations in AlN buffer and subsequent AlGaN heterostructures epitaxially grown on foreign substrates is one of the critical factors that limit the internal quantum efficiency (IQE) of the devices [4]. Therefore, extensive efforts have been made to improve the crystalline quality of the AlN buffer layers by developing special growth techniques [5]. For instance, a recent study reported the threading dislocation density (TDD) of the AlN buffer layer on sapphire (0001) as low as $2 \times 10^{8} \mathrm{~cm}^{-2}$ [6]. While this is a significant improvement, the approach has proven to result in only incremental improvement in cost/performance metric and to take significant resource in the epitaxial growth process. Furthermore, the reported TDD value is becoming closer to the state-of-the-art values of the GaN buffer on sapphire substrates for visible LEDs, which means that the further reduction in TDD of the buffer will be very challenging. Alternatively, the use of homoepitaxial growth on a native AIN substrate is more effective to further reduce the TDD [7]. However, the price of the native substrates is significantly higher and the size of the wafers is small, which has limited the adoption of the AlN substrates to only specialized high-end applications. Furthermore, the current AlN substrates show strong absorption bands in the UVC spectrum, which is detrimental to the emission of DUV LEDs [8].

We propose a new technological route to significantly improve the IQEs without resorting to the buffer layer or substrate material improvement. We exploit the mechanical flexibility of the semiconductor thin films to apply controlled external strain for the modification of electronic band structures of AlGaNbased heterostructures and the improvement of resulting device characteristics of the DUV LEDs. Bendable semiconductor LEDs have been demonstrated by the integration of a thin-film device structure on a flexible substrate [9-12]. The previous studies mainly focused on the integration of thin-film structures onto the flexible substrates and resulting mechanical flexibility for versatile applications of the LEDs on curved or variable surfaces, not on the effects of external strain on fundamental LED performance characteristics. 
The mechanical flexibility of the semiconductor thin films enables the application of high-degree external strain beyond the limit of internal lattice strain to the device structures. Especially with III-nitride (III-N) heterostructures under the influence of polarizations and resulting quantum-confined Stark effect (QCSE) [13], external strain can change the performance characteristics and provide additional functionality of the visible light emitters and transistors $[14,15]$. In this study, we show by numerical modeling that the change in the performance is particularly significant in DUV-LED structures due to unique properties of polarization coefficients and strain status of AlGaN heterostructures, as shown in table S1 of Supporting Information (SI).

\section{Device Modeling Details}

\subsection{Flexible DUV-LED device on bendable substrate}

Figure 1 shows a cross-sectional schematic of the flexible DUV-LED structure with an external bending load. The device structure experiences compressive and tensile strain in the bend-up (concave curvature, $R<0$ ) and bend-down (convex curvature, $R>0$ ) conditions, respectively, when a neutral plane is located in a flexible substrate. Figure S1 of SI shows the bending conditions and associated in-plane strains of the flexible LED structure. Typical DUV-LED chips have a flip-chip configuration with the $n$ side of the diode up toward a top emitting surface. Also, the substrate that was used for epitaxial growth should be removed for the flexibility of the LED structure. Therefore, we considered the fabrication process using the transfer of the flipped LED structure onto a flexible substrate, followed by the wafer substrate removal. The flexible substrates can be any flexible or curved-surface materials. Figure S2 of SI shows suggested fabrication steps to realize the flexible LED structure.

\subsection{DUV LED structure for simulation}

III-N heterostructures for the DUV LED consist of $\mathrm{Al}_{\mathrm{x}} \mathrm{Ga}_{1-\mathrm{x}} \mathrm{N}$ quantum wells (QWs) and $\mathrm{Al}_{\mathrm{y}} \mathrm{Ga}_{1-}$ ${ }_{y} \mathrm{~N}$ quantum-well barriers (QBs) sandwiched between an $\mathrm{Al}_{z} \mathrm{Ga}_{1-\mathrm{z}} \mathrm{N}$-based $p$ - $n$ junction on $\mathrm{AlN}$ buffer layer on top of a flexible substrate, as schematically shown in figure 1 . The buffer layer is $2-\mu$ m-thick with $\mathrm{Al}$ content graded $n-\mathrm{Al}_{\mathrm{x}} \mathrm{Ga}_{1-\mathrm{x}} \mathrm{N}$ from 1 to 0.5 with a doping concentration of $N_{D}=2 \times 10^{18} \mathrm{~cm}^{-3}$ by shallow donor $\mathrm{Si}\left(n-\mathrm{AlGaN}: \mathrm{Si}, 2 \mu \mathrm{m}, n \approx[\mathrm{Si}]=2 \times 10^{18} \mathrm{~cm}^{-3}\right)$. The DUV-LED structure with around $280 \mathrm{~nm}$ wavelength emission peak consists of an $\mathrm{Al}_{0.5} \mathrm{Ga}_{0.5} \mathrm{~N}$ layer $\left(n-\mathrm{Al}_{0.5} \mathrm{Ga}_{0.5} \mathrm{~N}: \mathrm{Si}, 1.3 \mu \mathrm{m}, n \approx[\mathrm{Si}]=2 \times 10^{18} \mathrm{~cm}^{-3}\right)$, five-period unintentionally doped QWs/QBs of $\mathrm{Al}_{0.42} \mathrm{Ga}_{0.58} \mathrm{~N} / \mathrm{Al}_{0.5} \mathrm{Ga}_{0.5} \mathrm{~N}(2.5 / 10 \mathrm{~nm})$, a $p-\mathrm{Al}_{0.7} \mathrm{Ga}_{0.3} \mathrm{~N}$ :Mg $(35 \mathrm{~nm}, p<$ $\left.[\mathrm{Mg}]=1 \times 10^{19} \mathrm{~cm}^{-3}\right)$ electron blocking layer $(\mathrm{EBL})$, a $p-\mathrm{Al}_{0.3} \mathrm{Ga}_{0.7} \mathrm{~N}: \mathrm{Mg}\left(p<[\mathrm{Mg}]=1 \times 10^{19} \mathrm{~cm}^{-3}\right)$, and a $p$ GaN:Mg $\left(100 \mathrm{~nm}, p<[\mathrm{Mg}]=5 \times 10^{19} \mathrm{~cm}^{-3}\right)$ contact layer. The EBL was used to reduce electron overflow out of active region. At room temperature, usually, a only few percent of $\mathrm{Mg}$ dopant in $p$-AlGaN ionizes and provides free hole carriers, $p$, as the activation energy of acceptor is very high, i.e., 170-470 meV [16]. A $p$-GaN layer was used for ohmic contact, although it absorbs a large portion of emitted photons which 
reduced light extraction efficiency (LEE). The electron and hole mobilities were assumed to be $100 \mathrm{~cm}^{2} \cdot \mathrm{V}^{-}$ ${ }^{1} \cdot \mathrm{s}^{-1}$ and $10 \mathrm{~cm}^{2} \cdot \mathrm{V}^{-1} \cdot \mathrm{s}^{-1}$, respectively [17]. Threading dislocation density for all layers was varied between $2 \times 10^{8} \mathrm{~cm}^{-2}$ and $1 \times 10^{10} \mathrm{~cm}^{-2}[18]$.

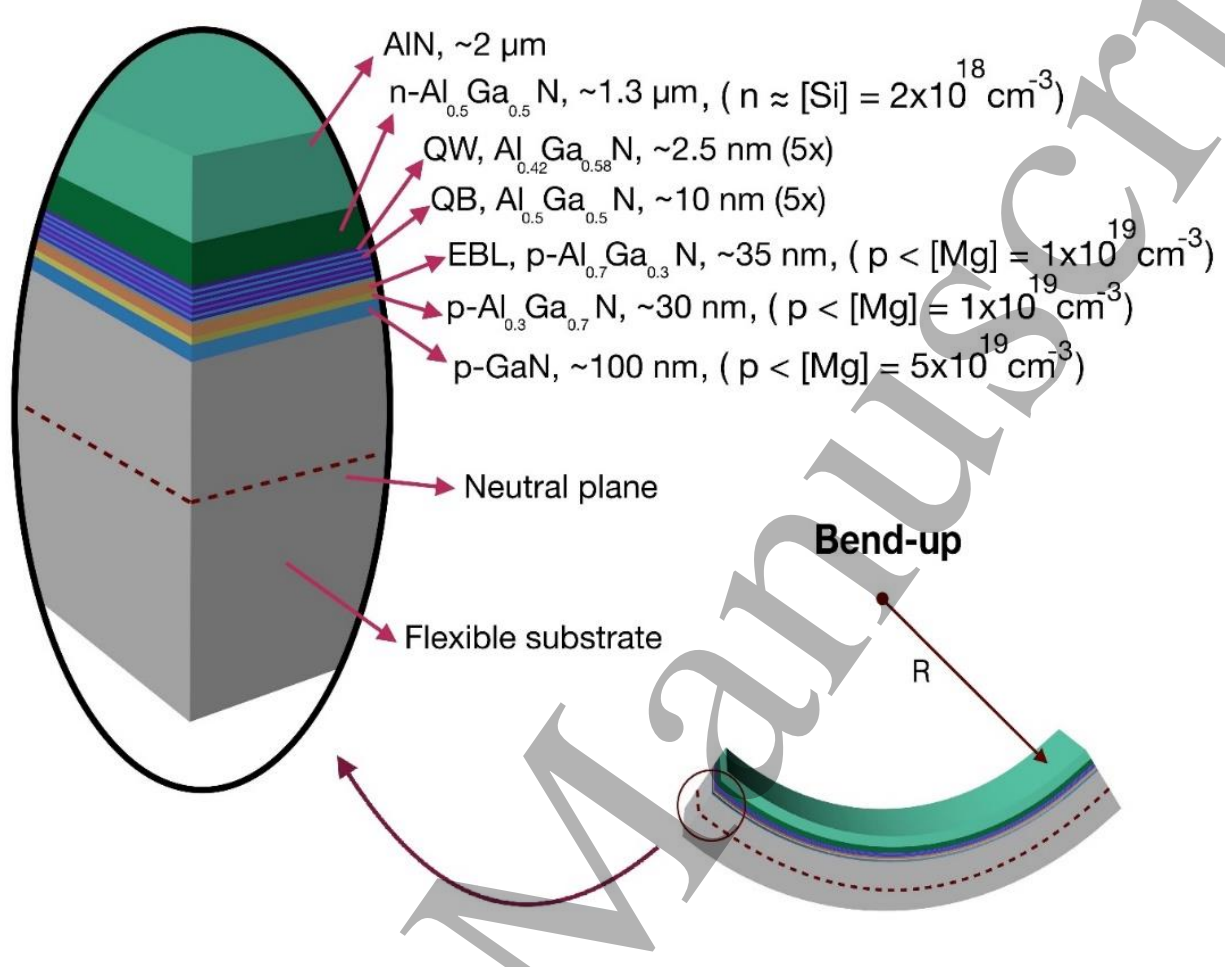

Figure 1. Schematic structure of layers (not to scale) of a deep-ultraviolet light-emitting diode (DUV LED) on a flexible substrate with a flip-chip thin-film configuration. An active region, consisting of AlGaN quantum well (QWs) and quantum-well barriers $(\mathrm{QBs})$ is sandwiched in a $p$ - $n$ junction with an electronblocking layer (EBL). Bend-up condition of a flexible structure is also illustrated.

\subsection{Applying external strain by bending for numerical studies}

The computational simulation was carried out by a commercial 1D technology computer-aided design (TCAD) device simulator [19], developed for the modeling of the operation of LED heterostructures based on direct-bandgap wurtzite semiconductors. The software simulates the band diagrams as a function of bias, electron and hole transport inside a heterostructure, and non-radiative and radiative carrier recombinations. The optical module employs Schrödinger equations for electrons and holes with the potential energy determined from a self-consistent solution of the Poisson and drift-diffusion transport equations. While many TCAD device simulators for photonic and electronic devices are available, they cannot be used in the calculation of flexible devices with active external loading. An additional module was developed in this study to include the effect of applied external bending strain on the polarization effects, quantum efficiency, and device characteristics. For the calculation of induced external strain by bending (figure S1 of SI), the isotropic elasticity theory was applied with assumptions of pure bending, no-slip 
boundary between layers, and linear mechanics [20]. Also, radially uniform distribution of strain in the flexible structures and unloaded edges of the substrate were assumed. Tensor was used to estimate strains $\left(\varepsilon_{x x}\left[=\eta_{a}\right], \varepsilon_{y y}, \varepsilon_{z z}, \varepsilon_{y z}, \varepsilon_{x z}, \varepsilon_{x y}\right)$ and resulting piezoelectric polarizations. We considered a biaxial strain condition for piezoelectric properties to retain the crystal symmetry of wurtzite structure under external load. Table S1 of SI shows important input parameters used in the simulation. The curvature radius $R$ was varied to consider various bending conditions in bend-up $(R<0)$ and bend-down $(R>0)$ modes as well as the no-bend condition $(R=\infty)$, and $L$ was set at $100 \mu \mathrm{m}$. The maximum applied strain on the structure was calculated as $\sim \pm 4 \%$ at $R= \pm 2.5 \mathrm{~mm}$.

\section{Results and Discussion}

The operation of LED includes carrier injection into the active region followed by photon generation by radiative recombination of the carriers (internal quantum efficiency [IQE, $\left.\eta_{I Q E}\right]$ ), and extraction of the photons from the device to free space (light-extraction efficiency [LEE, $\left.\eta_{L E E}\right]$ ). The overall efficiency, EQE, $\eta_{E Q E}$ is:

$$
\eta_{E Q E}=\eta_{I Q E} \times \eta_{L E E}
$$

The efficiency of each step is required to be maximized for the overall improved EQE. The IQE and LEE of state-of-the-art DUV LEDs are approximately $40 \%$ and $25 \%$, respectively [2]. Hence, there is significant potential for the IQE and LEE to be increased for the overall EQE improvement. Figure 2(a) shows the changes in IQE as a function of current density with different bending conditions. The overall IQE shifts to higher and lower values in bend-up and bend-down conditions, respectively, from the IQE in the no-bend condition. In the bend-up conditions, the IQE increases with increasing degree of bending (decreasing $|R|)$. Peak IQE increases from $27 \%\left(J=14 \mathrm{~A} / \mathrm{cm}^{2}\right)$ for the no-bend condition $(R=\infty)$ to $45 \%\left(J=80 \mathrm{~A} / \mathrm{cm}^{2}\right)$ and $65 \%\left(J=250 \mathrm{~A} / \mathrm{cm}^{2}\right)$ at $R=-5 \mathrm{~mm}$ and $-2.5 \mathrm{~mm}$, respectively. For the IQEs compared at the same current, e.g., at $J=200 \mathrm{~A} / \mathrm{cm}^{2}$, the IQE at $R=-2.5 \mathrm{~mm}(\sim 64 \%)$ is almost three time higher than that of the no-bend condition $(\sim 21 \%)$.

For the IQE change shown in figure 2(a), the TDD was assumed to be $\sim 2 \times 10^{8} \mathrm{~cm}^{-2}$, which is similar to the value reported by Shatalov, et al., where they have reported approximately two times improvement in IQE by decreasing the TDD from typical values of $\sim 1 \times 10^{9} \mathrm{~cm}^{-2}$ [6]. The improvement of the IQE occurs regardless of TDD, as show in figure 3. As the Shockley-Read-Hall (SRH) non-radiative recombination rate is strongly dependent on the defects, the IQE decreases with increasing TDD. Hence, the peak IQE decreases at higher TDDs. Nevertheless, the peak IQEs are improved significantly in the bend-up condition (red solid line in figure 3) as compared to those of no-bend conditions (black solid line in figure 3) in most TDD levels. Moreover, another advantage of this approach is highlighted at moderately high dislocation densities such as in $\sim 10^{9} \mathrm{~cm}^{-2}$ range. The peak IQEs for the bend-up mode $(R=-2.5 \mathrm{~mm})$ at TDD of $1 \times 10^{9}$ 
$\mathrm{cm}^{-2}$ and the no-bend mode at TDD of $2 \times 10^{8} \mathrm{~cm}^{-2}$ are $\sim 32 \%$ and $\sim 27 \%$ respectively. The IQE can be enhanced even higher by controlled external strain of the LED structure without any further development in growth process. For LED having the same structure with the TDD of $\sim 1 \times 10^{9} \mathrm{~cm}^{-2}$, the peak IQE can be improved more than six times from $\sim 5 \%$ in no-bend condition to $\sim 32 \%$ by applying external strain, which is more significant improvement in IQE than that by the TDD reduction to $\sim 2 \times 10^{8} \mathrm{~cm}^{-2}$.
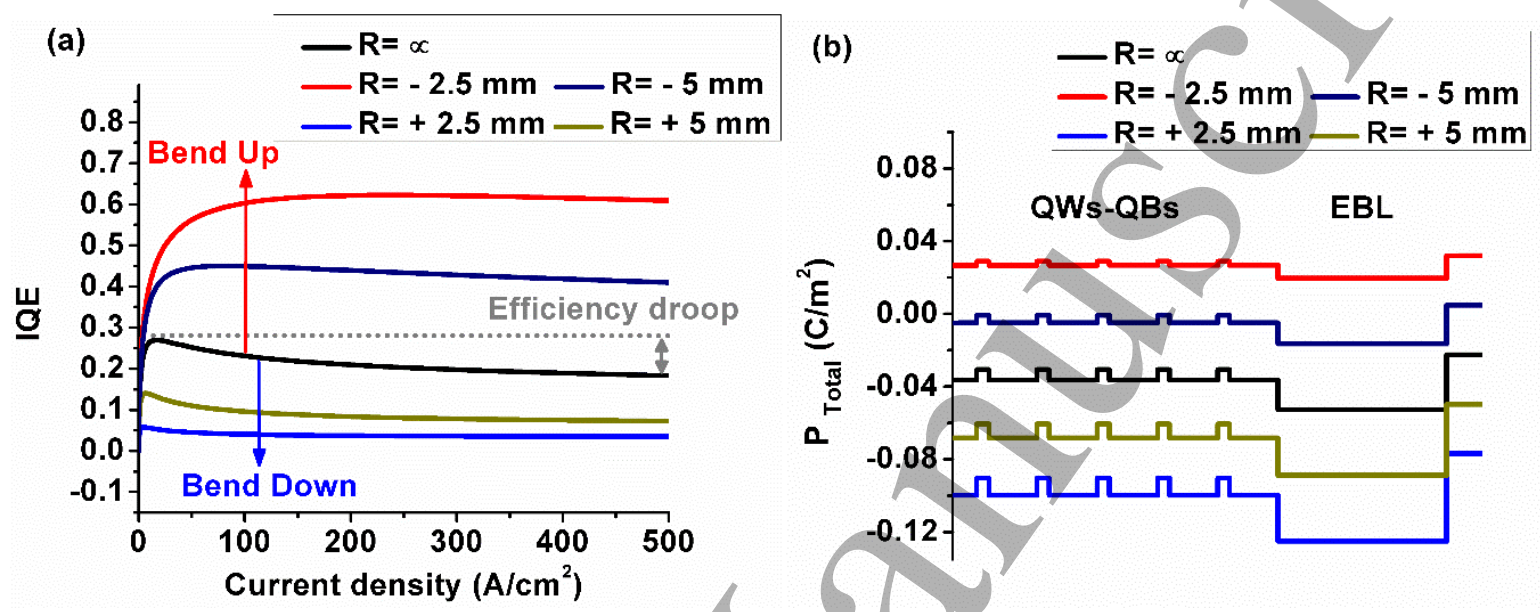

Figure 2. (a) Calculated internal quantum efficiencies (IQE) as a function of current density with different bending conditions. In a bend-up mode, IQE increases and efficiency droop is mitigated with increasing bending (decreasing $|R|$ ). (b) Calculated total polarization changes in an active region of QWs and QBs and an EBL in different bending conditions.

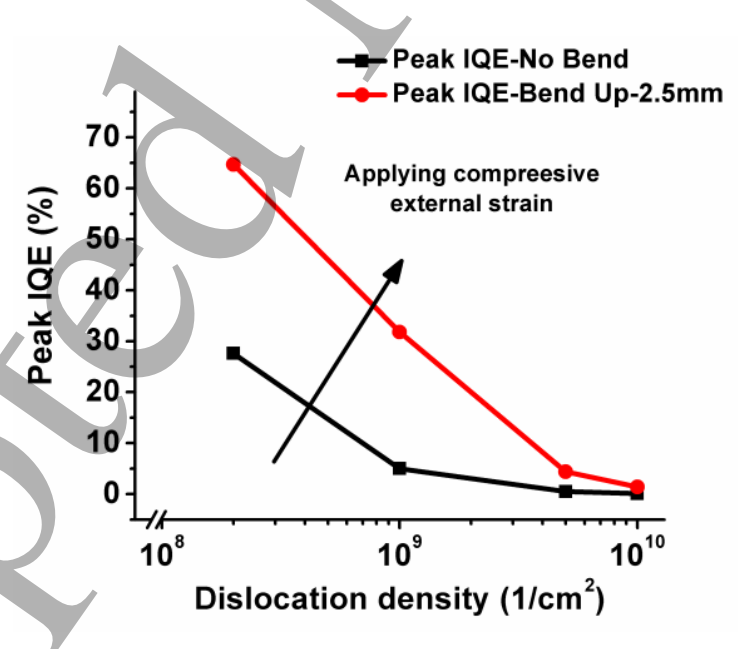

Figure 3. Calculated peak internal quantum efficiencies (IQE) as a function of dislocation density in bendup condition with $R=-2.5 \mathrm{~mm}$ and no-bend condition.

The degree of bending used in this study appears to be large. For experimental demonstration of bent LED structure, in fact, the degree of bending is limited by the maximum allowable mechanical flexibility. The limit is a strong function of the thickness and structure. However, a substantial degree of 
bending has been reported in semiconductor-based thin films with extremely small curvature radii of $\sim 265$ $\mathrm{nm}$ and $\sim 1.4 \mathrm{~mm}[21,22]$. The curvature radii we used in this study are significantly larger.

The IQE changes mainly originate from the modification of QCSE of AlGaN QW active region. The QCSE is another intrinsic limiting factor (besides crystalline defects) in the IQE of III-N-based devices. III-N materials, usually, are grown in a polar direction with gallium $(\mathrm{Ga})$-face along $c$-direction, forming a (0001) plane of wurtzite structure in which different types of polarization are involved [23, 24]. Both spontaneous and piezoelectric polarizations induce charges at the interfaces of the layers, where the induced polarization fields affect the electronic band structure and cause spatial separation in wave functions of electrons and holes; hence, carrier oscillator strength decreases [25]. Different approaches have been suggested to mitigate the polarization effects, including growing III-N structures on non-polar/semi-polar planes $[26,27]$ or controlling the degree of in-plane internally-induced piezoelectric polarization effect [28]. However, degree of freedom to modify the polarization effect was very limited. Active high-degree polarization control can be achieved by external bending. Figure 2(b) shows total polarization $\left(P_{\text {total }}\right)$ changes in an active region of QWs and QBs and an EBL in different bending conditions. Internal fields applied in the QWs originate from the difference in total polarization between QWs and QBs $\left(\Delta P_{\text {total }}\right)$. Therefore, the induced changes in $\Delta P_{\text {total }}$ is sensitive to polarization coefficients where applying external strain controls the piezoelectric polarization changes as well as the total polarization differences between QWs and QBs in different bending modes with various curvatures (figure S3 of SI). For the bend-up mode, applying compressive external strain induces the piezoelectric polarization that is anti-parallel to the existing total polarization (with negative sign); hence, the total polarization $\left(P_{\text {total }}\right)$ shifts toward positive values and $\Delta P_{\text {total }}$ decreases. The $\Delta P_{\text {total }}$ for bend-down (at $R=2.5 \mathrm{~mm}$ ), no-bend, and bend-up (at $R=-2.5$ $\mathrm{mm}$ ) modes are $\sim 0.01 \mathrm{C} / \mathrm{m}^{2}, \sim 0.006 \mathrm{C} / \mathrm{m}^{2}, \sim 0.002 \mathrm{C} / \mathrm{m}^{2}$, respectively. As discussed in SI (Equation S4 through S8), in the bend-up mode, the $\Delta P_{\text {total }}$ decreases by increasing bending and becomes nearly zero at $R=-2.5 \mathrm{~mm}$. The QCSE is mitigated in this condition, similar to the growth in a non-polar direction [29], in which polarization field is not induced inside QWs. In the bend-down mode, the QCSE becomes more significant with increasing bending. Therefore, the conduction and valence bands become almost flattened and more tilted for bend-up and bend-down modes, respectively. The spatial distribution of electrons and holes depends on the degree of tilt in the band diagram of QWs. In the bend-up mode, the overlap of electron and hole wave functions increases compared to that of the no-bend mode, as shown in figure 4(a). The oscillator strength of carriers and the consequent radiative recombination probability are enhanced. Therefore, a radiative recombination rate $\left(R_{r}\right)$ of electrons and holes in QWs increases, which results in IQE improvement in the bend-up mode. The IQE is defined as:

$$
\eta_{I Q E}=\eta_{\text {in }} \eta_{\text {rad }}=\eta_{\text {in }} \frac{R_{r}}{R_{r}+R_{n r}}
$$


where $\eta_{i n}$ is carrier injection efficiency, $\eta_{r a d}$ is radiative recombination efficiency, $R_{r}$ is a radiative recombination rate, and $R_{n r}$ is a non-radiative recombination rate. Non-radiative recombination includes SRH and Auger recombinations. While the degree of Auger recombination of III-N materials is still controversial, it is believed to be negligible in DUV LEDs due to low Auger recombination coefficient [30]. Hence, we assumed that only the SRH recombination, which is related to TDD, is responsible for the loss of injected carriers in a non-radiative way. Figure 3 also shows that the improvement by the bending becomes less effecive, when the dislocation densities are too high, e.g., $>1 \times 10^{10} \mathrm{~cm}^{-2}$, as the base-line IQE without bending is too low. When the rate of SRH recombination $\left(R_{n r}\right)$ becomes significantly high, the improvement of IQE from higher $R_{r}$ by the mitigation of QCSE becomes marginal.

(a)

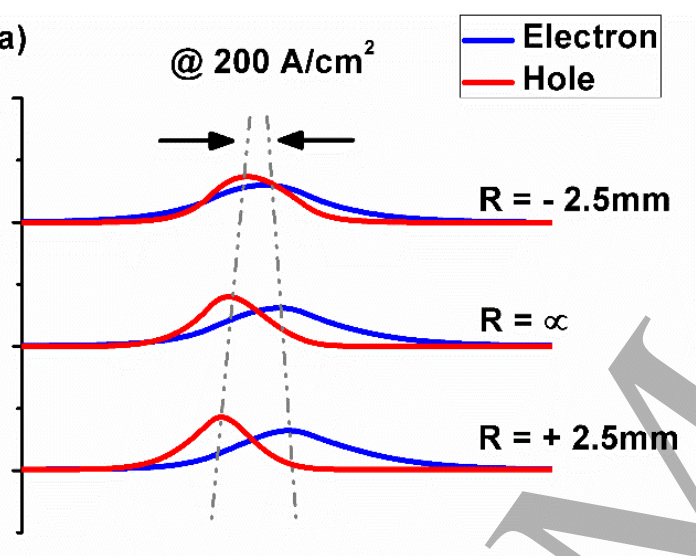

Figure 4. (a) Electron and hole wave functions in bend-up $(R=-2.5 \mathrm{~mm})$, no-bend, and bend-down $(R=$ $2.5 \mathrm{~mm}$ ) conditions for one quntum well at $J=200 \mathrm{~A} / \mathrm{cm}^{2}$. In bend-up mode, the wave functions of electron and hole are almost overlapped. (b) Cacluated electron leakage current density at the interface of EBL and active region. In bend-up mode $(R=-2.5 \mathrm{~mm})$, the electron loss to $p$-side is drastically decreased comparing to no-bend and bend-down modes.

Furthermore in figure 2(a), the IQE does not drop significantly in the bend-up mode. The high output power application of the DUV LEDs is limited by the efficiency droop [31]. Dominant mechanism of the efficiency droop in DUV LEDs is believed to be the electron spill-over from the MQWs to the $p$ $\mathrm{AlGaN}[32,33]$. Figure 5(a) compares the band diagrams of the active region and the EBL at $J=200 \mathrm{~A} / \mathrm{cm}^{2}$, where the difference in efficiency droop between no-bend and bend-up conditions is observed. The barrier height of the EBL for electron confinement is increased for the bend-up mode $(R=-2.5 \mathrm{~mm})$, as the conduction and valance bands of the EBL are shifted upward and more flattened. This is due to reduced $\Delta P_{\text {total }}$ between EBL and last QB, as shown in figure 2(b). In no-bend (and bend-down) conditions, however, electrons experience reduced height of the EBL, resulting in more electron leakage out of the active region, as shown in figure $4(b)$. 



Figure 5. (a) Calculated electronic band diagrams for an active region and an EBL in a DUV-LED structure in bend-up $(R=-2.5 \mathrm{~mm})$, no-bend $(R=\infty)$, and bend-down $(R=2.5 \mathrm{~mm})$ conditions at $J=200 \mathrm{~A} / \mathrm{cm}^{2}$. Inset shows barriers for electron injection (electron barriers). Barriers for electron confinement (EBL/active region barrier) and barriers for hole injection (hole barriers) are also displayed. (b) Calculated current density-voltage $(J-V)$ curves of a DUV-LED structure with various bending conditions (x-axis (bias) is broken in the range of $0-4 \mathrm{~V})$.

Figure 5(b) shows calculated current density vs. voltage $(J-V)$ curves in different bending conditions. By increasing the bending (decreasing $|R|)$ in the bend-up mode, the turn-on voltage decreases. Conversely, the turn-on voltage increases in the bend-down mode. This phenomenon is also related to the electronic band structure changes resulting from external strains. The barriers for electrons and holes to overcome at $J=200 \mathrm{~A} / \mathrm{cm}^{2}$ are shown in figure 5(a). The similar but higher barriers exist in equilibrium condition without applied bias, as shown in figure S4 of SI. For diode turn-on to flow the current through the $p$ - $n$ junction, electrons in $n$-AlGaN and holes in $p$-AlGaN are required to overcome the electron and hole barriers. In the bend-up mode, the barriers for electrons and holes, especially the hole barrier, decrease, resulting in lower turn-on voltage. In bend-down mode, the barriers are higher and higher voltage is required to maintain the same current density. This electrical property suggests that wall-plug efficiency (WPE) can be further improved in addition to the IQE improvement in the bend-up mode, since the device can be operated at lower voltage.

Another limiting factor in EQE of the DUV LEDs is inefficient extraction of generated photons. The LEE for further higher EQE is also expected to be improved in a curved semiconductor structures by increasing the angle of photon escape cones. The light extraction based on Monte Carlo ray tracing was carried out on the structure with a chip size of $700 \times 700 \mu \mathrm{m}^{2}$ in different bending conditions. The roughening of the photon-emission surface was not included intentionally to show the effect of the curved structure only. The LEE simulation results with relatively low LEE $<10 \%$ in the no-bend condition, which is typical for a single square LED chip. The LEE increases slightly by $\sim 0.5 \%$ in bent conditions, as shown in figure 
S5 of SI. This is due to the marginal increase in the angle of incidence of the cone due to the low curvature. While the low curvature is an advantageous characteristic for the IQE improvement without significant bending, it does not help expand the photon escape cone. In addition, top surface emission is not significant in dominant TM-mode emission in DUV LEDs [34]. Light distribution patterns show that the photon extraction is more efficient at the side-wall than the surface. Unlike the expectation, the increase in LEE for simulated bent LED structures is negligible. However, the flexible thin-film flip-chip LED structure in the bend-up condition with external compressive strain in QWs may offer another opportunity for LEE enhancement. The high degree compressive strain can change the optical polarization of photons from DUV QWs from TM dominant to TE dominant [35], which will enhance the vertical out-coupling of DUV light to top surface. This vertical out-coupling of photon in conjunction with the surface roughening of N-polar AIN surface is expected to significantly enhance the LEE with less detrimental effects from absorptive layers located underneath of the active region.

\section{Conclusions}

In conclusion, we showed that applying external bending changes the characteristics of the flexible DUV-LED devices. With controlled bending, (1) radiative recombination rate can be enhanced for higher IQEs; (2) hole transport and electron confinement are improved for mitigated efficiency droop; and (3) better electrical characteristics is achieved for higher WPE by lowering operating voltage of the diodes. The DUV LEDs can be integrated on a pre-defined curved packaging substrate to apply the controlled external strain. This will open an additional route for high-efficiency high-output-power applications of the DUV LEDs.

\section{Acknowledgments}

The work at University of Houston was supported in part by the Texas Center for Superconductivity at the University of Houston (TcSUH). The work at Hongik University was supported by the Basic Science Research Program through the National Research Foundation of Korea (NRF) funded by the Ministry of Education under grant 2015R1A6A1 A03031833. The work at Sunchon National University was supported by the Basic Science Research Program through the National Research Foundation of Korea (NRF) funded by the Ministry of Education under grant NRF-2015R1D1A3A01019050 and NRF-2014R1A6A1030419. XL appreciates the support of KAUST Baseline and Startup funding. 


\section{References}

\section{Uncategorized References}

[1] Kneissl M, Kolbe T, Chua C, Kueller V, Lobo N, Stellmach J, Knauer A, Rodriguez H, Einfeldt S, Yang Z, Johnson N M and Weyers M 2011 Advances in group III-nitride-based deep UV lightemitting diode technology Semiconductor Science and Technology 26014036

[2] Kneissl M and Rass J 2016 III-Nitride Ultraviolet Emitters,Technology and Applications (Springer International Publishing)

[3] Narukawa Y, Ichikawa M, Sanga D, Sano M and Mukai T 2010 White light emitting diodes with super-high luminous efficacy J. Phys. D-Appl. Phys. 436

[4] Shatalov M, Sun W, Jain R, Lunev A, Hu X, Dobrinsky A, Bilenko Y, Yang J, Garrett G A, Rodak L E, Wraback M, Shur M and Gaska R 2014 High power AlGaN ultraviolet light emitters Semiconductor Science and Technology 29084007

[5] Khan A, Balakrishnan K and Katona T 2008 Ultraviolet light-emitting diodes based on group three nitrides Nat. Photonics 2 77-84

[6] Shatalov M, Sun W, Lunev A, Hu X, Dobrinsky A, Bilenko Y, Yang J, Shur M, Gaska R, Moe C, Garrett G and Wraback M 2012 AlGaN Deep-Ultraviolet Light-Emitting Diodes with External Quantum Efficiency above 10\% Appl. Phys. Express 5082101

[7] Kim H J, Choi S, Yoo D, Ryou J-H, Dupuis R D, Dalmau R F, Lu P and Sitar Z 2008 Modulated precursor flow epitaxial growth of AlN layers on native AlN/substrates by metal-organic chemical vapor deposition Appl. Phys. Lett. 93

[8] Hartmann C, Wollweber J, Dittmar A, Irmscher K, Kwasniewski A, Langhans F, Neugut T and Bickermann M 2013 Preparation of Bulk AlN Seeds by Spontaneous Nucleation of Freestanding Crystals Japanese Journal of Applied Physics $\mathbf{5 2}$

[9] Jung Y, Wang X, Kim J, Kim S H, Ren F, Pearton S J and Kim J 2012 GaN-based light-emitting diodes on origami substrates Appl. Phys. Lett. 100

[10] Kim R-H, Kim D-H, Xiao J, Kim B H, Park S-I, Panilaitis B, Ghaffari R, Yao J, Li M, Liu Z, Malyarchuk V, Kim D G, Le A-P, Nuzzo R G, Kaplan D L, Omenetto F G, Huang Y, Kang Z and Rogers J A 2010 Waterproof AlInGaP optoelectronics on stretchable substrates with applications in biomedicine and robotics Nature Materials 9 929-37

[11] Kim S H, Singh S, Oh S K, Lee D K, Lee K H, Shervin S, Asadirad M, Venkateswaran V, Olenick K, Olenick J A, Lee S-N, Kwak J S, Mavrokefalos A and Ryou J-H 2016 Visible Flip-Chip LightEmitting Diodes on Flexible Ceramic Substrate With Improved Thermal Management IEEE Electron Device Lett. 37 615-7

[12] Sher C-W, Chen K-J, Lin C-C, Han H-V, Lin H-Y, Tu Z-Y, Tu H-H, Honjo K, Jiang H-Y, Ou SL, Horng R-H, Li X, Fu C-C and Kuo H-C 2015 Large-area, uniform white light LED source on a flexible substrate Opt. Express 23 A1167-A78

[13] Ryou J-H, Yoder P D, Liu J, Lochner Z, Kim H, Choi S, Kim H J and Dupuis R D 2009 Control of Quantum-Confined Stark Effect in InGaN-Based Quantum Wells IEEE J. Sel. Topics Quantum Electron. 15 1080-91

[14] Shervin S, Kim S-H, Asadirad M, Karpov S Y, Zimina D and Ryou J-H 2016 Bendable III-N Visible Light-Emitting Diodes beyond Mechanical Flexibility: Theoretical Study on Quantum Efficiency Improvement and Color Tunability by External Strain ACS Photonics 3 486-93

[15] Shervin S, Kim S-H, Asadirad M, Ravipati S, Lee K-H, Bulashevich K and Ryou J-H 2015 StrainEffect Transistors: Theoretical Study on the Effects of External Strain on III-Nitride High-ElectronMobility Transistors on Flexible Substrates Appl. Phys. Lett. 107193504

[16] Nakarmi M L, Nepal N, Lin J Y and Jiang H X 2009 Photoluminescence studies of impurity transitions in Mg-doped AlGaN alloys Appl. Phys. Lett. 94091903

[17] Mnatsakanov T T, Levinshtein M E, Pomortseva L I, Yurkov S N, Simin G S and Khan M A 2003 Carrier Mobility Model for GaN Solid-State Electron. 47 111-5 
[18] Cooke M 2014 Deep UV LED research moving performance beyond 10\% efficiency vol 9 (Semiconductor Today )

[19] www.str-soft.com/products/SimuLED www.str-soft.com/products/SimuLED.

[20] Boresi A P and Schmidt R J 2002 Advanced Mechanics of Materials, 6th Edition (Wiley)

[21] Schmidt O G and Eberl K 2001 Nanotechnology - Thin solid films roll up into nanotubes Nature $410168-$

[22] Kim J, Hwang J, Song K, Kim N, Shin J C and Lee J 2016 Ultra-thin flexible GaAs photovoltaics in vertical forms printed on metal surfaces without interlayer adhesives Appl. Phys. Lett. 1085

[23] Miller D A B, Chemla D S, Damen T C, Gossard A C, Wiegmann W, Wood T H and Burrus C A 1984 Band-Edge Electroabsorption in Quantum Well Structures - The Quantum-Confined StarkEffect Physical Review Letters 53 2173-6

[24] Bernardini F and Fiorentini V 1999 Spontaneous versus Piezoelectric Polarization in III-V Nitrides: Conceptual Aspects and Practical Consequences. Physica Status Solidi B-Basic Research 216391 8

[25] Im J S, Kollmer H, Off J, Sohmer A, Scholz F and Hangleiter A 1998 Reduction of Oscillator Strength due to Piezoelectric Fields in GaN/AlxGa1-xN Quantum Wells Phys. Rev. B 57 R9435R8

[26] Gardner N F, Kim J C, Wierer J J, Shen Y C and Krames M R 2005 Polarization Anisotropy in the Electroluminescence of m-plane InGaN-GaN Multiple-Quantum-Well Light-Emitting Diodes Appl. Phys. Lett. 863

[27] Sharma R, Pattison P M, Masui H, Farrell R M, Baker T J, Haskell B A, Wu F, DenBaars S P, Speck J S and Nakamura S 2005 Demonstration of a Semipolar (101-3-) InGaN/GaN Green Light Emitting Diode Appl. Phys. Lett. 873

[28] Aumer M E, LeBoeuf S F, Bedair S M, Smith M, Lin J Y and Jiang H X 2000 Effects of Tensile and Compressive Strain on the Luminescence Properties of AlInGaN/InGaN Quantum Well Structures Appl. Phys. Lett. 77 821-3

[29] Waltereit P, Brandt O, Trampert A, Grahn H T, Menniger J, Ramsteiner M, Reiche M and Ploog K H 2000 Nitride Semiconductors Free of Electrostatic Fields for Efficient White Light-Emitting Diodes Nature 406 865-8

[30] Delaney K T, Rinke P and Van de Walle C G 2009 Auger recombination rates in nitrides from first principles Appl. Phys. Lett. 943

[31] Sun W, Shatalov M, Deng J, Hu X, Yang J, Lunev A, Bilenko Y, Shur M and Gaska R 2010 Efficiency droop in 245-247 nm AlGaN light-emitting diodes with continuous wave $2 \mathrm{~mW}$ output power Appl. Phys. Lett. 96061102

[32] Choi S, Ji M-H, Kim J, Jin Kim H, Satter M M, Yoder P D, Ryou J-H, Dupuis R D, Fischer A M and Ponce F A 2012 Efficiency droop due to electron spill-over and limited hole injection in IIInitride visible light-emitting diodes employing lattice-matched InAlN electron blocking layers Appl. Phys. Lett. 101161110

[33] Yun J, Shim J-I and Hirayama H 2015 Analysis of efficiency droop in 280-nm AlGaN multiplequantum-well light-emitting diodes based on carrier rate equation Appl. Phys. Express 8022104

[34] Lee K H, Park HJ, Kim S H, Asadirad M, Moon Y T, Kwak J S and Ryou J H 2015 Light-extraction efficiency control in AlGaN-based deep-ultraviolet flip-chip light-emitting diodes: a comparison to InGaN-based visible flip-chip light-emitting diodes Opt Express 23 20340-9

[35] Northrup J E, Chua C L, Yang Z, Wunderer T, Kneissl M, Johnson N M and Kolbe T 2012 Effect of strain and barrier composition on the polarization of light emission from AlGaN/AlN quantum wells Appl. Phys. Lett. 100 